\title{
Hypokalemia secondary to capecitabine: a hidden toxicity?
}

\author{
Muhammad Wasif Saif ${ }^{\prime}$ \\ Mohammad Houman \\ Fekrazad $^{2}$ \\ Leslie Ledbetter ${ }^{3}$ \\ Robert B Diasio ${ }^{3}$ \\ 'Yale University School of Medicine, \\ New Haven, CT; ${ }^{2}$ Carraway \\ Methodist Medical Center, \\ Birmingham, AL; ${ }^{3}$ University of \\ Alabama at Birmingham, AL, USA \\ Presented at the Annual Meeting of \\ ASCO, Orlando, FL in 2005.
}

Correspondence: M Wasif Saif Yale University School of Medicine, Section of Medical Oncology, 333 Cedar Street; FMP II6,

New Haven, CT 06520, USA

Tel +| 203737 |875

$\mathrm{Fax}+\mathrm{I} 2037853788$

Email wasif.saif@yale.edu
Background: Hyopkalemia is a listed toxicity in the capecitabine (Xeloda ${ }^{\circledR}$; Roche, Nutley, NJ) package insert. However, the incidence and severity of this toxicity is not known.

Methods: We performed a retrospective evaluation of hypokalemia in 77 patients, who received capecitabine for gastrointestinal malignancies between April 2002 and November 2004. Hypokalemia was defined as $\mathrm{K}^{+}$level $<3.2 \mathrm{mEq} / \mathrm{L}$. Patients with documented $\geq$ grade 2 vomiting or diarrhea, diuretics, hypomagnesemia, hypokalemia, renal insufficiency, endocrine dysfunction (thyroid, adrenal, diabetic) were excluded. Hypokalemic patients were graded as: mild (grade 1: 3.0-3.2 mEq/L), moderate (grade 3: 2.5-2.9 mEq/L) and severe (grade 4: $<2.5 \mathrm{mEq} / \mathrm{L}$ ). We also reviewed the literature.

Results: Fifty-four patients met the above criteria. The most common cause of exclusion was $\geq$ grade 2 diarrhea (23 patients; 30\%). Overall, hypokalemia was encountered in 11 patients $(20.4 \%)$. Among hypokalemic patients, 8 patients (73\%) presented with mild/grade 1 hypokalemia (3.0$3.2 \mathrm{mEq} / \mathrm{L}), 2$ patients $(18.18 \%)$ with moderate/grade 3 hypokalemia $(2.5-2.9 \mathrm{mEq} / \mathrm{L})$ and 1 patient $(9.09 \%)$ with severe/grade 4 hypokalemia $(<2.5 \mathrm{mEq} / \mathrm{L}) 8(73 \%)$. Dose of capecitabine ranged between 1000-2000 mg/m². Hypokalemia occurred after an average of 83.7 days of capecitabine administration. No cardiac or neuromuscular complications were noticed. Replacement of $\mathrm{K}^{+}$was required in 6 patients ( 2 intravenous and 4 oral), while 2 patients (3.7\%) required oral supplements $>4$ weeks. No patient had to stop capecitabine due to hypokalemia. One patient had persistent hypokalemia even after stopping capecitabine. Normalization of $\mathrm{K}^{+}$levels was achieved in $91 \%$ of patients. Four patients were on $\mathrm{K}^{+}$sparing diuretics for ascites and never presented with hypokalemia. Mean urine $\mathrm{K}^{+}$was $28 \mathrm{mEq} / \mathrm{L}$. Only $5.5 \%$ patients had $\geq$ grade 3 hypokalemia in our study compared with $2 \%$ and $14 \%$ in two other studies.

Conclusions: Although diarrhea being the most common cause of hypokalemia in patients on capecitabine, we postulate that hypokalemia may also be related to the effect of capecitabine on renal tubules suggested by the urine $\mathrm{K}^{+}$in some patients. Due to potential complications, hypokalemia in patients on capecitabine deserves special diagnostic and therapeutic attention.

Keywords: 5-FU, capecitabine (Xeloda), hypokalemia, renal loss, potassium, colon cancer

\section{Introduction}

Capecitabine (Xeloda ${ }^{\circledR}$; Roche, Nutley, NJ) is a tumor-activated antineoplastic agent (antimetabolite) belonging to the novel fluoropyrimidine carbamate class (FDA 2001). Capecitabine is currently indicated for the first-line treatment of patients with metastatic colorectal carcinoma when treatment with fluoropyrimidine therapy alone is preferred (Hoff et al 2001; Van Cutsem et al 2001). It is also indicated in combination with docetaxel for the treatment of patients with metastatic breast cancer after failure of prior anthracycline containing chemotherapy (O'Shaugnessy et al 2002) and as monotherapy for the treatment of patients with metastatic breast cancer resistant to both paclitaxel and an anthracycline-containing chemotherapy or resistant to paclitaxel, but with contraindications to anthracyline therapy (Blum et al 1999). The studies in these cancer populations have shown evidence that capecitabine provides an effective alternative to current standard intravenous chemotherapy and in some 
instances superior efficacy in treatment of metastatic colorectal and breast cancer (Twelves 2002). Capecitabine allows patients to avoid the placement of long-term intravenous access which provides two major benefits: fewer complications associated with intravenous catheters and more freedom for the patient during the treatment process. Capecitabine is currently being evaluated for use in combination with radiotherapy and other chemotherapeutic agents such as oxaliplatin, irinotecan, and many others.

The most common side effects of capecitabine include diarrhea, nausea, vomiting, stomatitis, abdominal pain, handand-foot syndrome, and fatigue. These side effects are similar to what one would expect in using fluoropyrimidines. However, when compared with intravenous bolus infusion of 5-FU/LV (Mayo Regimen) in these phase III studies, the incidence of nausea, diarrhea, and alopecia were significantly lower in the patients receiving capecitabine (Twelves 2002). The patients receiving capecitabine also had lower incidences of grade 3 to 4 stomatitis and neutropenia resulting in less frequent hospitalizations. Incidences of fatigue and vomiting were similar in both arms of the study. Capecitabine was associated with more frequent occurrences of hand-foot syndrome, but this can be successfully managed by dose reduction and prevention. The most significant laboratory abnormality found in the capecitabine arm compared with 5-FU arm was elevation of total bilirubin. Elevated total bilirubin was found in $21 \%$ of patients receiving capecitabine versus only $2 \%$ of patients receiving 5-FU/Leucovorin (Twelves 2002).

Less frequent but serious complications of capecitabine include cardiotoxicity, and neurotoxicity (Saif et al 2003, 2004). Hypokalemia has not been reported frequently with capecitabine. We encountered three patients who developed hypokalemia in the absence of diarrhea, vomiting, or other etiologies. This prompted us to perform a retrospective study to determine incidence, risk factors, complications, and management of hypokalemia in patients receiving capecitabine. To systematically estimate the frequency of this complication of capecitabine therapy, we obtained a waiver of authorization from the institutional review board (IRB) of University of Alabama at Birmingham (UAB) to review the laboratory and pharmacy profiles for all gastrointestinal (GI) malignancy patients treated with capecitabine.

\section{Patients and methods}

Retrospective review of all patients with GI malignancies who were treated with capecitabine alone or capecitabinebased regimen by one of the oncologists from April 2002 and November 2004 at the UAB was conducted after approval from the IRB. A total of 77 patients were identified with a final diagnosis of cancer arising in the pancreas, colon, rectum, liver, and stomach. Neither clinical trials nor standard practice guidelines mandated routine monitoring of potassium levels.

Exclusion criteria included presence of documented $\geq$ grade 2 (NCI-CTC 2.0) vomiting or diarrhea, concomitant use of diuretics or antibiotics (eg, penicillin, gentamicin, amphotericin), hypomagnesemia, history of hypokalemia prior to capecitabine administration, renal insufficiency, thyroid (hypothyroidism) or adrenal dysfunction (Addison's) and diabetic nephropathy. Out of 77 patients, 54 were eligible.

The data including age, sex, race, initial weight, final diagnosis, dose and date of capecitabine administration, concurrent diuretics, radiation (XRT) or other chemotheraputic agents, diabetes mellitus, hypothyroidism, and renal dysfunction were recorded. We also collected the laboratory results on serum creatinine, potassium, magnesium, and urine potassium in these patients. Most of these patients had threeweekly laboratory data collection, while after noticing hypokalemia, serum electrolytes were followed weekly till resolution of the abnormality. The urine electrolytes, in particular urine $\mathrm{K}^{+}$were available only in patients who were worked up for hypokalemia. Patients charts were reviewed to evaluate for any cardiac (symptoms or electrocardiogram [EKG] changes) or neuromuscular complications due to hypokalemia. Also information about potassium replacement was collected up to a month after stopping capecitabine.

\section{Statistical analysis}

Hypokalemia was defined as $\mathrm{K}^{+}$level $<3.2 \mathrm{mEq} / \mathrm{L}$. Patients were classified into 3 groups based on $\mathrm{K}^{+}$levels: (1) mild/ grade $1(3.0-3.2 \mathrm{mEq} / \mathrm{L}),(2)$ moderate/grade $3(2.5-2.9 \mathrm{mEq} / \mathrm{L})$ and, (3) severe/grade $4(<2.5 \mathrm{mEq} / \mathrm{L})$. Baseline serum and urine values (if available) were compared with values obtained after capecitabine administration. For subjects with more than one urine sample within an interval, the mean of the individual samples was used. Correlation between serum creatinine and potassium was analysed. All data were recorded in Microsoft Excel 98 (Microsoft Corporation; Seattle, WA); all statistical analysis was performed using SAS (version 8.2; SAS Institute; Cary, NC).

\section{Results}

Among 77 patients identified, 54 patients met the eligibility criteria as described above. They were 30 male and 24 female 
patients with median age of 60 years (range: 32-89). The final diagnoses included: pancreatic cancer (31), colon cancer (19), hepatocellular carcinoma (3), and gastric cancer (1) (Table 1). The most common reason for exclusion was diarrhea $\geq$ grade 2 ( 23 patients; $30 \%$ ). Twenty patients received capecitabine monotherapy $(37 \%): 5$ patients received capecitabine alone and 15 patients received capecitabine with concomitant XRT followed by capecitabine monotherapy (all pancreatic cancer patients who received this regimen as a part of a clinical trial); and 34 patients in combination with other chemotherapeutic agents (63\%). These agents included oxaliplatin and irinotecan, both not known to cause hypokalemia. The dose of capecitabine ranged between $1000-2000 \mathrm{mg} / \mathrm{m}^{2}$. No correlation between the dose of capecitabine and hypokalemia was observed in these patients.

Among 54 patients who received capecitabine and met our criteria, 11 patients $(20.4 \%)$ had hypokalemia. Of those, 8 patients (73\%) presented with mild/grade 1 hypokalemia (3.0-3.2 $\mathrm{mEq} / \mathrm{L}), 2$ patients (18.18\%) with moderate/grade 3 hypokalemia $(2.5-2.9 \mathrm{mEq} / \mathrm{L})$ and 1 patient $(9.09 \%)$ with severe/grade 4 hypokalemia $(<2.5 \mathrm{mEq} / \mathrm{L})$ (Table 2$)$. No patient developed any cardiac (EKG) or neuromuscular complications due to hypokalemia. Diagnosis of

Table I Demographic data of patients with gastrointestinal malignancies on capecitabine

\begin{tabular}{|c|c|}
\hline & Total $=54^{*}$ \\
\hline Gender $=$ male: female & $(54)=30: 24$ \\
\hline Age: Median (range) & 60 years $(32-89)$ \\
\hline Ethnicity = White: Black & $50: 4$ \\
\hline \multicolumn{2}{|l|}{ Diagnosis } \\
\hline Pancreatic cancer & 31 \\
\hline Colorectal cancer & 19 \\
\hline Hepatocellular cancer & 3 \\
\hline Gastric cancer & 1 \\
\hline \multicolumn{2}{|l|}{ Chemotherapy regimens } \\
\hline Capecitabine monotherapy & 5 \\
\hline Capecitabine $+\mathrm{XRT}$ & 15 \\
\hline Capecitabine + other chemotherapic agent(s) & 34 \\
\hline $\begin{array}{l}\text { Average days since Xeloda at time of } \\
\text { hypokalemia }\end{array}$ & 84 days \\
\hline \multicolumn{2}{|l|}{ Grades of hypokalemia } \\
\hline Mild/Grade I hypokalemia (3.0-3.2 mEq/L) & 8 \\
\hline Moderate/Grade 3 hypokalemia $(2.5-2.9 \mathrm{mEq} / \mathrm{L})$ & 2 \\
\hline Severe/Grade 4 hypokalemia (<2.5 mEq/L) & 1 \\
\hline Mean urine potassium & $\begin{array}{l}28 \mathrm{mEq} / \mathrm{L} \\
\text { (range: } 2 \mathrm{I}-5 \mathrm{I} \text { ) }\end{array}$ \\
\hline
\end{tabular}

Note: $* 54$ out of 77 patients met the criteria. hypokalemia occurred after an average of 83.7 days of capecitabine administration. Six patients $(11 \%)$ required replacement of $\mathrm{K}^{+}: 2$ patients received intravenous $\mathrm{K}^{+} ; 4$ patients were administered oral $\mathrm{K}^{+}$). Continued administration of oral supplements of $\mathrm{K}^{+}>4$ weeks was required in 2 patients (3.7\%). One patient (1.8\%) had persistent hypokalemia even after stopping capecitabine. None of our patients had to stop capecitabine due to hypokalemia. Normalization of potassium levels was achieved in $91 \%$ of patients. Four patients $(7.4 \%)$ were on $\mathrm{K}^{+}$sparing diuretics (spironolactone) for ascites and never presented with hypokalemia.

No correlation in serum creatinine and hypokalemia was noticed. However, decrease in serum $\mathrm{K}^{+}$was proportional to the amount of urine $\mathrm{K}^{+}$(Table 2). Mean urine $\mathrm{K}^{+}$was $28 \mathrm{mEq} / \mathrm{L}$ (range: $21-51 \mathrm{mEq} / \mathrm{L}$ ), suggesting renal loss of potassium in these patients.

\section{Discussion}

No previous case reports relating an association between capecitabine use and the occurrence of hypokalemia are found in the literature. The capecitabine package insert does list that hypokalemia is a potential toxicity under capecitabine monotherapy in breast and colorectal cancer (Roche Pharmaceuticals 2001), but the real incidence or the severity of this side effect is not known. We found hypokalemia in $>20 \%$ of patients on capecitabine without any obvious etiology, including severe diarrhea or vomiting or the use of loop diuretics. Approximately $5.5 \%$ patients had $\geq$ grade 3 hypokalemia. The incidence of hypokalemia might be higher as few patients $(7.4 \%)$ were on $\mathrm{K}^{+}$sparing diuretics. No correlation between hypokalemia and dosage of capecitabine was observed. However, there was an apparent relation between the serum $\mathrm{K}^{+}$and urine $\mathrm{K}^{+}$levels. Due to the potential complications, hypokalemia in patients on capecitabine deserves special diagnostic and therapeutic attentions. Further analysis to characterize the mechanism is needed.

The most common reason for exclusion was diarrhea $\geq$ grade 2 (23 patients; $30 \%$ ). This finding is akin to the observed incidence of diarrhea in major studies which evaluated capecitabine such as those performed by Van Custem et al (2001) and Hoff et al (2001). However, we did not find any information about hypokalemia in both studies. In the study done by Hoff et al (2001), 8 (2.7\%) patients required in-patient re-hydration. In the study done by Van Custem et al (2001), 5 (1.7\%) patients required in-patient rehydration. We reviewed studies as well as other major trials on the pub med. In the study by Cassidy and colleagues

Therapeutics and Clinical Risk Management 2007:3(I) 
Table 2 Correlation of hypokalemia with other factors

\begin{tabular}{|c|c|c|c|}
\hline Total = I I & $\begin{array}{l}\text { Mild/Grade I } \\
\text { hypokalemia }(\mathrm{N}=8)\end{array}$ & $\begin{array}{l}\text { Moderate/Grade } 3 \\
\text { hypokalemia }(\mathrm{N}=2)\end{array}$ & $\begin{array}{l}\text { Severe/Grade } 4 \\
\text { hypokalemia }(\mathrm{N}=1)\end{array}$ \\
\hline Serum creatinine $(\mathrm{mg} / \mathrm{dl})$ mean (range) & $0.97(0.4-1.1)$ & $0.85(0.6-1.1)$ & 0.6 \\
\hline Urine potassium (mEq/L) mean (range) & $21(18-28)$ & $36(3 I-36)$ & $5 I^{*}$ \\
\hline Level of $\mathrm{Mg}$ at low potassium $(\mathrm{mg} / \mathrm{dl})$ & 1.65 & 1.5 & 2.0 \\
\hline Capecitabine dose $\left(1000 \mathrm{mg} / \mathrm{m}^{2}\right)$ & 2 & 1 & 0 \\
\hline Capecitabine dose $\left(2000 \mathrm{mg} / \mathrm{m}^{2}\right)$ & 6 & I & I \\
\hline
\end{tabular}

Note: *Available in only one patient.

(2002) comparing capecitabine versus 5-FU in metastatic colorectal cancer mentioned the incidence of laboratory abnormalities, including liver enzymes and blood count, but no data is available regarding potassium in the published paper. In a phase II study done by Jatoi and colleagues (2006) evaluating oxaliplatin and capecitabine in patients with metastatic adenocarcinoma of the esophagus, gastroesophageal junction, and gastric cardia, 6 (out of 43) patients developed $\geq$ grade 3 hypokalemia (14\%). In our study, of the 11 patients who developed hypokalemia: 2 were on oxaliplatin with capecitabine; 1 was on irinotecan with capecitabine; and the remaining 8 were on capecitabine monotherapy. Both these agents (oxaliplatin, irinotecan) are not known to cause hypokalemia. However, we suggest that the interaction of all these combinations need to be looked at as confounding factors.

We suggest that, like several other important oncology drugs such as cisplatin, which is known to be associated with excessive loss of magnesium and potassium in the urine (Rodriguez et al 1989), capecitabine may also impair potassium reabsorption in the kidney and may cause clinically significant hypokalemia. We suggest that similar cases of hypokalemia associated with capecitabine should be reported if observed. Spot urine potassium might be a helpful diagnostic test in detection of the source of potassium loss in these patients. However, a 24-hour collection of urine for potassium would yield better information.

The pitfalls of retrospective study are well known, such as lack of complete information about the toxicities and the absence of urine $\mathrm{K}^{+}$in all the hypokalemic and normokalemic patients, but some conclusions may be drawn from this study. Our study showed that late onset hypokalemia was encountered in $>20 \%$ of patients on capecitabine. Most of the patients (73\%) presented with grade 1 hypokalemia, and $18.18 \%$ with grade 3 hypokalemia and $9 \%$ with grade 4 hypokalemia. It is reassuring that no significant clinical problems were found in the patients in this study. We believe that our findings on hypokalemia in this study are intriguing and suggest the need of more research on this topic. Management of hypokalemia in patients on capecitabine deserves special attention due to the potential complications. This issue is also significant in patients who are already on diuretics. Further analysis will be required to characterize the mechanism for the apparent renal potassium wasting in these patients.

\section{References}

Blum JL, Jones SE, Buzdar AU, et al. 1999. Multicenter phase II study of capecitabine in paclitaxel-refractroy metastatic breast cancer. J Clin Oncol, 17:485-93.

Cassidy J, Twelves C, Van Cutsem E, et al.; Capecitabine Colorectal Cancer Study Group. 2002. First-line oral capecitabine therapy in metastatic colorectal cancer: a favorable safety profile compared with intravenous 5-fluorouracil/leucovorin. Ann Oncol, 13: 566-75.

[FDA] Food and Drug Administration. 2001. Xeloda package insert [online]. Accessed on 23 May 2006. URL: http://www.fda.gov/ cder/foi/label/2000/20896lbl.pdf.

Hoff PM, Ansari R, Batist G, et al. 2001. Comparison of oral capecitabine versus intravenous fluorouracil plus leucovorin as first-line treatment in 605 patients with metastatic colorectal cancer: results of a randomized phase III study. J Clin Oncol, 19:2282-92.

Jatoi A, Murphy BR, Foster NR, et al. 2006. Oxaliplatin and capecitabine in patients with metastatic adenocarcinoma of the esophagus, gastroesophageal junction and gastric cardia: a phase II study from the North Central Cancer Treatment Group. Ann Oncol, 17:29-34.

O'Shaugnessy J, Miles D, Vukelja S, et al. Superior survival with capecitabine plus docetaxel combination therapy in athracyclinepretreated patients with advanced breast cancer: phase III trial results. J Clin Oncol, 20:2812-23.

Rodriguez M, Solanki DL, Whang R. 1989. Refractory potassium repletion due to Cisplatin-induced magnesium depletion. Arch Intern Med, 149:2592-4.

Saif MW, Quinn MG, Thomas RR, et al. 2003. Cardiac toxicity associated with capecitabine therapy. Acta Oncol, 42:342-4.

Saif MW, Wood TE, McGee PJ, et al. 2004. Peripheral neuropathy associated with capecitabine. Anticancer Drugs, 15:767-71.

Twelves C. 2002. Capecitabine as first-line treatment in colorectal cancer: pooled data from two large, phase III trials. Eur J Cancer, 38:S15-20.

Van Cutsem E, Twelves C, Cassidy J et al. 2001. Oral capecitabine compared with intravenous fluorouracil plus leucovorin in patients with metastatic colorectal cancer: results of a large phase III study. $J$ Clin Oncol, 19:4097-106. 